
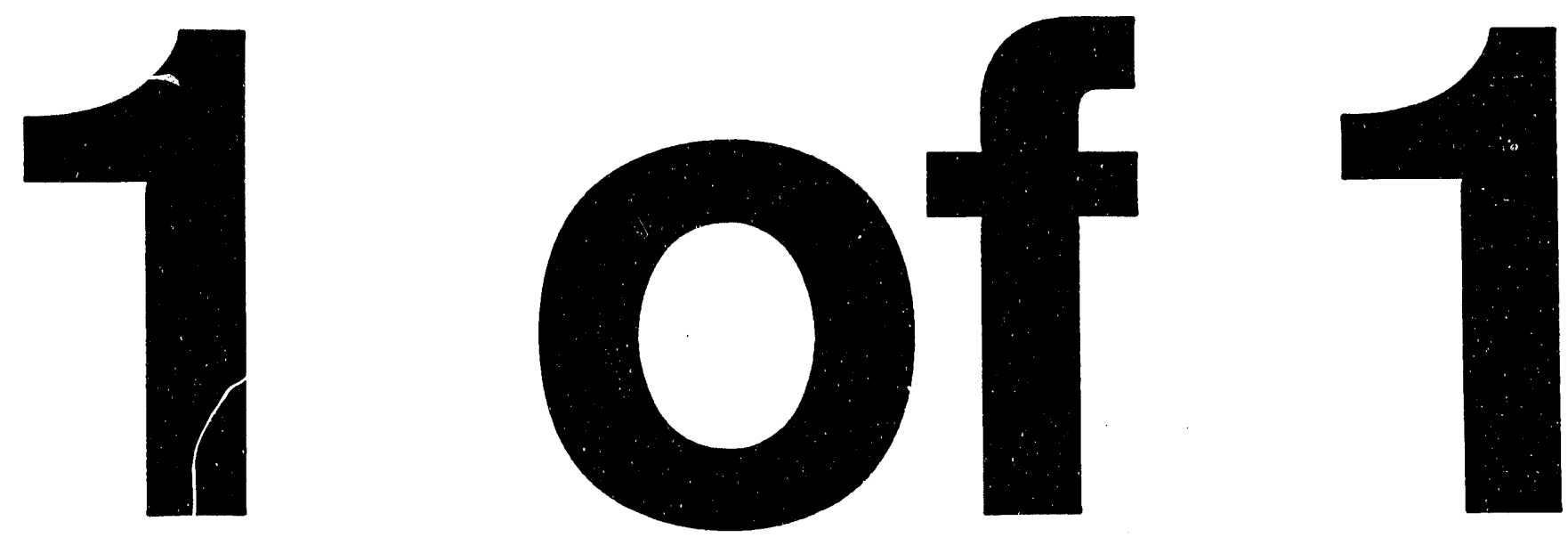
UCRL-JC-115131

PREPRINT

\title{
Model-Based Inversion for a Shallow Ocean Application
}

\author{
J. V. Candy \\ E. J. Sullivan
}

This paper was prepared for submittal to the Inversion Methods in Ocean and Seismic Acoustics Conference

La Spezia, Italy

June 27, 1994

March 1994

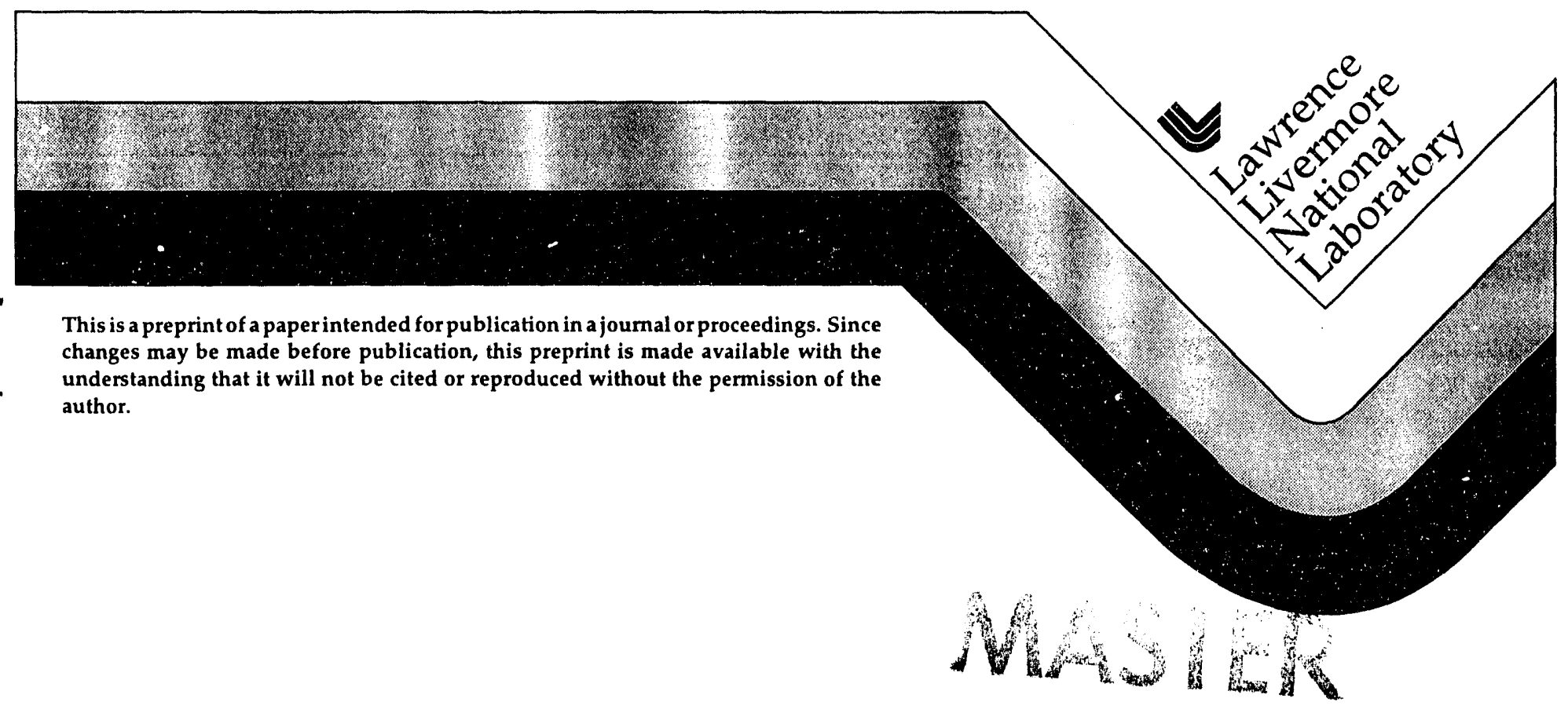




\section{DISCLAIMER}

This document was prepared as an account of work sponsored by an agency of the United States Government. Neither the United States Government nor the University of California nor any of their employees, makes any warranty, express or implied, or assumes any legal liability or responsibility for the accuracy, completeness, or usefulness of any information, apparatus, product, or process disclused, or represents that its use would not infringe privately owned rights. Reference herein to any specific commercial products, process, or service by trade name, trademark, manufacturer, or otherwise, does not necessarily constitute or imply its endorsement, recommendation, or favoring by the United States Government or the University of California. The views and opinions of authors expressed herein do not necessarily state or reflect those of the United States Government or the Lniversity of Califormia, and shall not be used for advertising or product endorsement purposes. 
MODEL-BASED INVERSION

FOR A SHALLOW OCEAN APPLICATION

\author{
J.V. CANDY \\ Lawrence Livermore National Laboratory \\ P.O. Box 808, L-495 \\ Livermore, CA 94550
}

\author{
E.J. SULLIVAN \\ Naval Undersea Warfare Center \\ NUSC Code 103 \\ Newport, RI 02841
}

ABSTRACT. A model-based approach to invert or estimate the sound speed profile (SSP) from noisy pressure-field measurements is discussed. The resulting model-based processor (MBP) is based on the state-space representation of the normal-mode propagation model. Using data obtained from from the well-known Hudson Canyon experiment, a noisy shallow water ocean environment, the processor is designed and the results compared to those predicted using various propagation models and data. It is shown that the MBP not only predicts the sound speed quite well, but also is able to simultaneously provide enhanced estimates of both modal and pressure-field measurements which are useful for localization and rapid ocean environmental characterization.

\subsection{Introduction}

The rapid assessment of environmental conditions is extremely important in a tactical as well as experimental situation. The incorporation of ocean acoustic propagation models into signal processing schemes has long been the hope to improve overall processor performance. Current applications are concerned with the localization/detection of an acoustic source as well as inversion and the proposed methods are based on bringing the predictions of a propagation model in concert with pressure measurements from an array. This is called the matched-field problem and much work has been accomplished [1-4]. Model-based techniques offer high expectations of performance, since a processor based on the predicted physical phenomenology that inherently has generated the measured signal must produce a better (minimum variance) estimate than one that does not [5]. However, if the model embedded in this processor is inaccurate or for that matter incorrect, than the model-based processor (MBP) can actually perform worse. Therefore, it is necessary, as part of the MBP design procedure, to estimate the model parameters either through separate experiments or jointly (adaptively) while performing the required processing $[6,7]$. Note that the introduction of the recursive, on-line MBP can offer a dramatic detection improvement in a tactical passive or active sonar-type system especially when a rapid environmental assessment is required.

In ocean acoustics we are usually concerned with an environmental model of the ocean and how it effects the propagation of sound through this noisy, hostile environment. The problem of estimating the environmental parameters characterizing the ocean medium is called the ocean tomography or equivalently the environmental inversion problem and has long been a concern because of its deterimental effect on various detection/localization schemes $[8,9]$. Much of the work accomplished on this problem has lacked quantitative measures of the mismatch of the model with its environment. In a related work [10] it was shown how to quantify "modeling errors" both with a known ocean environmental model available and without. In the first case, it was shown how to calculate standard errors for modal/pressure-field estimates as well as an overall measure of fit based on the innovations or residuals between the measured and predicted pressure-field. In the second case, only the residuals are used. These results quantify the mismatch between the embedded models and the actual measurements both on simulated as well as experimental data.

In this paper we concentrate on the design of a MBP to solve the environmental inversion or oceanographic tomography problem while jointly estimating the underlying signals - which we term model-based inversion. That is, the MBP is designed to estimate the sound speed profile (SSP) as well as enhance the corresponding modal/pressure-field signals with its accompanying performance statistics quantified using both an established model and the corresponding residuals. More specifically, we are concerned with whether or not it is possible to estimate the SVP from noisy

This work was performed under the auspices of the Department of Energy by the Lawrence Livermore National Laboratory under contract W-7405-Eng-48. 
hydrophone measurements in a real, hostile ocean acoustic experimental environment. Theoretical work on the design of MBP for this problem has been accomplished recently indicating that a solution exists, howe ver, most of the results presented were theoretical and supported by a simple shallow water simulation [7]. Here, in contrast, we actually apply these techniques for inversion to measured data from the Hudson Canyon experiment - a well-known and substantiated area for such an application. Note that there is presently a growing literature on oceanographic tomography, since it can deal with the estimation of many different parameters relative to ocean acoustics. For more information on these issues, see $[8,9]$ and references therein.

We briefly review the state-space propagator of the normal mode model for the Hudson Canyon experiment which will be used in this paper in sec. 2 . In the next section we design a model-based processor to estimate the SVP from noisy experimental hydrophone measurements for this shallow water, ocean acoustic problem.

\subsection{State Space Propagators for Hudson Canyon}

In this section we discuss the development of the propagators for the Hudson Canyon experiment performed in 1988 in the Atlantic and led by W. Carey of Advanced Research Projects Agency with the primary goal of investigating acoustic propagation (transmission and attenuation) using continuous wave data. The Hudson Canyon is located off the coast of New Jersey in the area of the Atlantic Margin Coring project borehole 6010. The seismic and coring data are combined with sediment properties measured at that site. Excellent agreement was determined between the model and data indicating a well-known, well-documented shallow water experiment with bottom interaction and yielding ideal data sets for investigating the applicability of a MBP to measured ocean acoustic data [11]. The experiment was performed at low frequencies $(50-600 \mathrm{~Hz})$ in shallow water of $73 \mathrm{~m}$ depth during a period of calm sea state. A calibrated acoustic source was towed at roughly $36 \mathrm{~m}$ depth along the $73 \mathrm{~m}$ isobath radially to distances of 4 to $26 \mathrm{Km}$. The ship speed was between 2 and $4 \mathrm{kts}$. The fixed vertical hydrophone array consisted of 24 phones spaced $2.5 \mathrm{~m}$ apart extending from the seafloor up to a depth of about $14 \mathrm{~m}$ below the surface. CTD and SSP measurements were made at regular intervals and the data were collected under carefully controlled conditions in the ocean environment. The normalized horizontal wave number spectrum for a $50 \mathrm{~Hz}$ temporal frequency is dominated by 5 modes occurring at wave numbers between 0.14 to $0.21 \mathrm{~m}^{-1}$ with relative amplitudes increasing with increased wave number. A SNAP [12] simulation was performed and the results agree quite closely, indicating a well-understood ocean environment.

In order to construct the state-space propagator, we require the set of parameters which were obtained from the experimental measurements and processing (wave number spectra). The horizontal wave number spectra were estimated using synthethic aperture processing [13]. Eight temporal frequencies were employed: four on the inbound $(75 \mathrm{~Hz}, 275 \mathrm{~Hz}, 575 \mathrm{~Hz}, 600 \mathrm{~Hz})$ and four on the outbound $(50 \mathrm{~Hz}, 175 \mathrm{~Hz}, 375 \mathrm{~Hz}, 425 \mathrm{~Hz})$. In this application we will confine our investigation to the $50 \mathrm{~Hz}$ case, which is well-documented, and to horizontal ranges from $0.5-4 \mathrm{Km}$. The raw measured data was processed (sampled, corrected, filtered, etc.) by J. Doutt of the Woods Hole Oceanographic Institute and supplied for this investigation.

For this study we assume a horizontally-stratified ocean of depth $h$ with a known source position $(x, y)$ and assume that the acoustic energy from a point source can be modeled as a trapped wave governed by the wave equation. We obtain the solution to this equation following the approach of Pekeris [14] leading to the well-known normal-mode equations.

A solution of the range dependent relation is given by a Hankel function and the depth relation is an eigenvalue equation in $z$ with

$$
\frac{d^{2}}{d z^{2}} \phi_{m}(z)+\kappa_{z}(m) \phi_{m}(z)=0, m=1, \cdots, M
$$

whose eigensolutions $\left\{\phi_{m}(z)\right\}$ are the so called modal functions and $\kappa_{z}$ is the wavenumber in the z-direction. These solutions depend on the sound speed profile, $c(z)$, and the boundary conditions 
at the surface and bottom as well as the corresponding dispersion relation.

The normal-mode solutions can easily be placed in state-space form and we refer the interested reader to Refs. $[6,7]$ for the detailed theory. Our approach for the Hudson Canyon experiment is to place the model into a Gauss-Markov representation which includes the second order statistics. The measurement noise can represent the near-field acoustic noise field, flow noise on the hydrophone and electronic noise. The modal noise can represent SSP errors, distant shipping noise, errors in the boundary conditions, sea state effects and ocean inhomogeneities. By assuming that the horizontal range of the source $r_{\theta}$ is known a-priori, we can use the Hankel function $H_{0}\left(\kappa_{r} r_{n}\right)$ which is the source range solution; therefore, we reduce the state-space model to that of "depth only" and the Gauss-Markov model for this constrained problem is given by

$$
\frac{d}{d z} \mathbf{x}(z)=A(z) \mathbf{x}(z)+B(z) \mathbf{u}(z)+\mathbf{w}(z)
$$

with the pressure field measurement model given by

$$
p(z)=\mathbf{C}^{T}\left(r_{s}, z\right) \mathbf{x}(z)+\mathbf{v}(z)
$$

where

$$
\mathbf{C}^{T}\left(r_{s}, z\right)=\left[\begin{array}{lll}
\beta_{1}\left(r_{s}, z\right) & 0|\cdots| \beta_{M}\left(r_{s}, z\right) & 0
\end{array}\right]
$$

with $\beta_{i}\left(r_{s}, z\right)=\frac{q \phi_{i}\left(z_{s}\right)}{\int_{0}^{h} \phi_{i}^{2}(z) d z} H_{0}\left(k_{r}(i) r_{s}\right)$. The random noise vectors $\underline{w}$ and $\underline{v}$ are assumed gaussian, zero-mean with respective covariance matrices, $R_{w w}$ and $R_{v v}$.

Since our array spatially samples the pressure-field, we choose to discretize the differential state equations using a finite (first) difference approach, that is, for the $m^{\text {th }}$-mode we use

$$
\frac{d}{d z} \underline{x}_{m}\left(z_{\ell}\right) \approx \frac{\underline{x}_{m}\left(z_{\ell+1}\right)-\underline{x}_{m}\left(z_{\ell}\right)}{\Delta z_{\ell}}
$$

where $\Delta z_{\ell}=z_{\ell+1}-z_{\ell}$. Therefore, substituting into Eqn. 2, we obtain

$$
\underline{x}_{m}\left(z_{\ell+1}\right)=\left[I+\Delta z_{\ell} A_{m}\left(z_{\ell}\right)\right] \underline{x}_{m}\left(z_{\ell}\right)+\Delta z_{\ell} \underline{B}\left(z_{\ell}\right) u\left(z_{\ell}\right)+\underline{w}\left(z_{\ell}\right)
$$

Assuming we have a vertical line sensor array to measure the pressure-field, the measurement model for the $m^{\text {th }}$ mode becomes

$$
p_{m}\left(r_{s}, z_{\ell}\right)=\underline{C}_{m}^{T}\left(r_{s}, z_{\ell}\right) \underline{x}_{m}\left(z_{\ell}\right)+v_{m}\left(z_{\ell}\right)
$$

The vertical wave numbers are functions of the sound speed profile through the dispersion relationship and can be further analyzed through knowledge of the SSP. Since our processor will be sequential, it is recursing over depth. We would like our processor to improve the estimation of the SSP "in-between" sensors. With a state-space processor we can employ two spatial increments in z simultaneously: one for the measurement system $\Delta z_{\ell}$ and one for the modal state-space $\Delta z_{j}=\frac{\Delta z_{\ell}}{N_{\Delta}}$ where $N_{\Delta}$ is an integer. Therefore in order to propagate the states (modes) at $\Delta z_{j}$ and measurements at $\Delta z_{\ell}$ we must have values of the SSP at each $\Delta z_{j}$ (subinterval) as well. Suppose we expand $c(z)$ in a Taylor series about a nominal depth, say $z_{0}$, then we obtain

$$
c(z) \approx \theta_{0}\left(z_{0}\right)+\theta_{1}\left(z_{0}\right)\left(z-z_{0}\right)+\cdots+\theta_{N}\left(z_{0}\right) \frac{\left(z-z_{0}\right)^{N}}{N !}
$$

where $\theta_{i}\left(z_{0}\right):=\left.\frac{\partial^{i} c(z)}{\partial z^{i}}\right|_{z=z_{0}} \quad i=0, \cdots, N$. In this formulation we therefore have a "model" of the SSP of the form $\hat{c}_{N}\left(z_{\ell}\right)=\underline{\Delta}_{N}^{T}\left(z_{\ell}\right) \underline{\theta}\left(z_{\ell}\right)$ where the set of $\left\{\theta_{i}\left(z_{\ell}\right)\right\}$ are only known at a sparse number 
of depths, $\ell=j, j+1, \cdots, j+N_{\theta}$. More simply, we have a set of measurements of the SSP measured a-priori or on-line at specific depths - not necessarily corresponding to all sensor locations $\left\{z_{\ell}\right\}$, therefore, we use these values as initial values to the MBP enabling it to sequentially update the set of parameters $\left\{\theta_{i}\left(z_{j}\right)\right\}$ over the layer $z_{\ell-1} \leq z_{j}<z_{\ell}$ until a new value of $\theta_{i}\left(z_{j}\right)$ becomes available, then we re-initialize the parameter estimator with this value and continue our SSP estimation until we have recursed through each sensor location. In this way we can utilize our measured SSP information in the form of a parameter update and improve the estimates using the processor. Thus, we can characterize this SSP representation in an approximate Gauss-Markov model which is nonlinear when we constrain the SSP parameters to the set $\left\{\theta\left(z_{\ell}\right)\right\}, z_{\ell}=z_{j}, \cdots, z_{j}+N_{\theta}$, that is,

$$
\underline{\theta}_{N}\left(z_{\ell+1}\right)= \begin{cases}\underline{\theta}_{N}\left(z_{\ell}\right)+\Delta z_{\ell} \underline{w}_{\theta}\left(z_{\ell}\right) & z_{\ell}<z_{j}<z_{\ell+1} \\ \underline{\theta}_{N}\left(z_{\ell}\right) \delta\left(z_{\ell}-z_{j}\right) & z_{\ell}=z_{j}\end{cases}
$$

where we have $w_{\theta} \sim N\left(0, R_{w_{\theta} w_{\theta}}\right), \underline{\theta}_{N}\left(z_{0}\right) \sim N\left(\underline{\theta}_{N}(0), P_{\theta}(0)\right)$, and $\triangle_{N}^{T}\left(z_{\ell}\right)=\left[1 \quad \Delta z_{\ell-1} \ldots \frac{x_{\ell-1}^{N}}{N \mid}\right]$.

It is this model that we use in our MBP to estimate the sound speed and solve the environmental inversion problem. We can now "augment" this SSP representation into our normal-mode/pressurefield propagation model to obtain an overall system model. The augmented Gauss-Markov (approximate) model for $M$-modal functions in the interval $z_{\ell}<z_{j}<z_{\ell+1}$ is given by

$$
\left[\begin{array}{c}
\underline{x}\left(z_{\ell+1}\right) \\
-\underline{\theta}_{N}\left(z_{\ell+1}\right)
\end{array}\right]=\left[\begin{array}{ccc}
A\left(z_{\ell}, \theta\right) & \mid & 0 \\
\overline{0} & - & - \\
I_{N+1}
\end{array}\right]\left[\begin{array}{c}
\underline{x}\left(z_{\ell}\right) \\
\hdashline \underline{\theta}_{N}\left(z_{\ell}\right)
\end{array}\right]+\left[\begin{array}{c}
B\left(z_{\ell}\right) \\
-\overline{0}- \\
\underline{u}
\end{array}\right] \underline{u}(z)+\left[\begin{array}{c}
\underline{w}\left(z_{\ell}\right) \\
\hline-\underline{w_{\theta}\left(z_{\ell}\right)}
\end{array}\right]
$$

with corresponding measurement model

$$
p\left(r_{s}, z_{\ell}\right)=\left[\begin{array}{lll}
\underline{C}^{T}\left(r_{s}, z_{\ell}\right) & \mid \underline{0}
\end{array}\right]\left[\begin{array}{c}
\underline{x}\left(z_{\ell}\right) \\
-\underline{\theta}\left(z_{\ell}\right)
\end{array}\right]+v_{p}\left(z_{\ell}\right)
$$

This completes the development of the state-space propagator for the experiment, next we discuss the design of the MBP for the Hudson Canyon data.

\subsection{Model-Based Inversion: Sound Speed Estimator}

In this section we develop a solution to the environmental inversion problem by designing a MBP to estimate the sound speed, on-line, from noisy pressure-field measurements. The processor is based on the state-space representation of the normal-mode model and a vertical line array of sensors along with the augmented model discussed in the previous section. We briefly discuss the approach, then the algorithm and apply it to the Hudson Canyon experimental data for a $500 \mathrm{~m}$ range at a $50 \mathrm{~Hz}$ temporal frequency. The environment inversion problem can be defined in terms of our previous models as: GIVEN a set of noisy acoustic (pressure-field) measurements $\left\{p\left(r_{0}, z_{\ell}\right)\right\}$ and a set of sound speed parameters $\left\{\underline{\theta}\left(z_{\ell}\right)\right\}$, FIND the "best" (minimum variance) estimate of the SSP, $\hat{c}\left(z_{\ell}\right)$. For this problem we have a sparse set of SSP measurements available at $N_{\theta}=9$ depths with complete set of pressure-field measurements. The solution to the inversion problem can be obtained using the extended Kalman filter (EKF) algorithm or its variants [5] as a joint state/parameter estimator. Here we choose discrete EKF available in SSPACK_PC [15].

The experimental measurements consists of sound speed in the form of discrete data pairs $\left\{c\left(z_{j}\right), z_{j}\right\}$ which can be utilized in the estimator for correction as it processes the acoustic data. We use the first two terms $(\mathrm{N}=2)$ of the Taylor series expansion of the SSP (piecewise linear) for our model where both $\theta_{0}$ and $\theta_{1}$ are space-varying gaussian random functions with specified means 
and variances, and therefore, through linearity, so is $c\left(z_{\ell}\right)$. Thus, our Gauss-Markov model for this problem is given by Eqns. 10 and 11. We will use a spatial sampling interval of $\Delta z_{j}=\frac{\Delta z_{f}}{10}$ in the state propagation equations as discussed previously. The corresponding EKF estimator evolves from the algorithm (see [7]) with all of the appropriate functions and jacobians.

We observe the performance of the model-based SSP processor. Here we use only the acoustic measurements and the 9 sound speed data values $\left\{c\left(z_{j}\right)\right\}, j=1, \cdots, 9$ to set hard constraints on the parameter estimator and force it to meet these values only when the appropriate depth is achieved. The results of the runs are shown in Figure 1. Here we see the estimated SSP parameters and reconstructed SSP. The estimator appears to track the SSP parameters as well as the profile. The standard rms modeling errors (see [10] for details) for the SSP parameters and profile are respectively: $1.6 \times 10^{-4} ; 2.7 \times 10^{-5} ; 1.0 \times 10^{-2}$. Since we are using a joint estimator, the enhanced estimates of both modal functions and the pressure field are shown in Figure 2 along with the corresponding mean predicted using the SNAP model parameters. Here we see excellent agreement with all of the modes (except mode 1). The standard rms modeling errors for each mode, respectively, are: $1.0 \times 10^{-2} ; 1.3 \times 10^{-3} ; 2.3 \times 10^{-4} ; 2.2 \times 10^{-4} ; 3.5 \times 10^{-4}$. The innovations or residuals are shown in Figure 3 where we see that they are zero mean and reasonably white (8.3\% out of bounds). The rms standard error for the residuals is given by $2.9 \times 10^{-3}$. So we see that the processor is clearly capable of jointly estimating the SSP and enhancing the modal/pressure-field.

\subsection{Discussion}

In this paper we have developed an on-line, adaptive, model-based solution to the evironmental inversion problem, that is, a sound speed profile estimation scheme based on coupling the normalmode propagation model to a functional model of the SSP evolving from Taylor series expansion about the most current sound speed measurement available. The algorithm employed was the extended Kalman filter which evolved as the solution to the minimum variance estimation problem when the inodels were placed in state-space form. With the inavailability of complete set of sound speed measurements, we showed that the processor performs reasonably well even for very sparse sound speed information.

\section{References}

[1] Hinich, M.J. (1973) Maximum likelihood signal processing for a vertical array, J. Acoust. Soc. Am., 54, 499-503.

[2] Bucker, H.P. (1976) Use of calculated sound fields and matched-field detection to locate sound in shallow water, J. Acoust. Soc. Am., 59, 329-337.

[3] Sullivan, E.J. and Middleton, D. (1993) Estimation and detection issues in matched-field processing, IEEE Trans. Ocean Eng., 18, (3), 156-167.

[4] Baggeroer, A.B., Kuperman, W.A., and Schmidt, H. (1988) Matched-field processing: source localization in correlated noise as an optimum parameter estimation problem, J. Acoust. Soc. $A m, 83,(2), 571-587$.

[5] Candy, J. V. (1986) Signal Processing: The Model-Based Approach. New York:McGraw-Hill.

[6] Candy, J.V. and Sullivan, E.J. (1992) Ocean acoustic signal processing: a model-based approach, J. Acoust. Soc. Am., 92, 3185-3201.

[7] Candy, J.V. and Sullivan, E.J. (1993) Sound velocity profile estimation: a system theoretic approach, IEEE Trans. Ocean Eng., 18, (3), 240-252.

[8] Tolstoy, A. (1992) Review of matched field processing for environmental inverse problems, Int. J. Modern Physics, 3, (4), 691-708. 
[9] Tolstoy, A. (1993) Matched Field Processing for Ocean Acoustics. New Jersey:World Scicntific Publishing.

[10] Candy, J.V. and Sullivan, E.J. (1994) Model-based processor design for a shallow water ocean acoustic experiment, J. Acoust. Soc. Am., 95, (4) 1-14.

[11] Rogers, A.R., Yamamoto, Y. and Carey, W. (1993) Experimental investigation of sediment effect on acoustic wave propagation in shallow water, J. Acoust. Soc. Am., 93,1747-1761.

[12] Jensen, F.B. and Ferla, M.C. (1982) SNAP: the SACLANTCEN normal-mode acoustic propagation model," SACLANTCEN Report, SM-121, SACLANT Undersea Research Centre, La Spezia, Italy.

[13] Sullivan, E.J., Carey, W. and Stergiopoulos, S., Eds. (1993) Special Issue on Acoustic Synthetic Aperture Processing, IEEE Trans. Ocean Eng., 17.

[14] Clay, C. S. and Medwin, H. (1977) Acoustical Oceanography. New York:Wiley.

[15] Candy, J.V. and Candy, P.M. (1993) SSPACK_PC: A model-based signal processing package on personal computers, DSP Applic., 2,(3), 33-42.

\section{Acknowledgements}

We would like to acknowledge the support of Dr. R. Doolittle of the Office of the Chief of Naval Research (OCNR) under the auspices of the High Gain Initiative and Dr. N. Gerr of OCNR under the Ocean Acoustic Signal Processing Initiative.
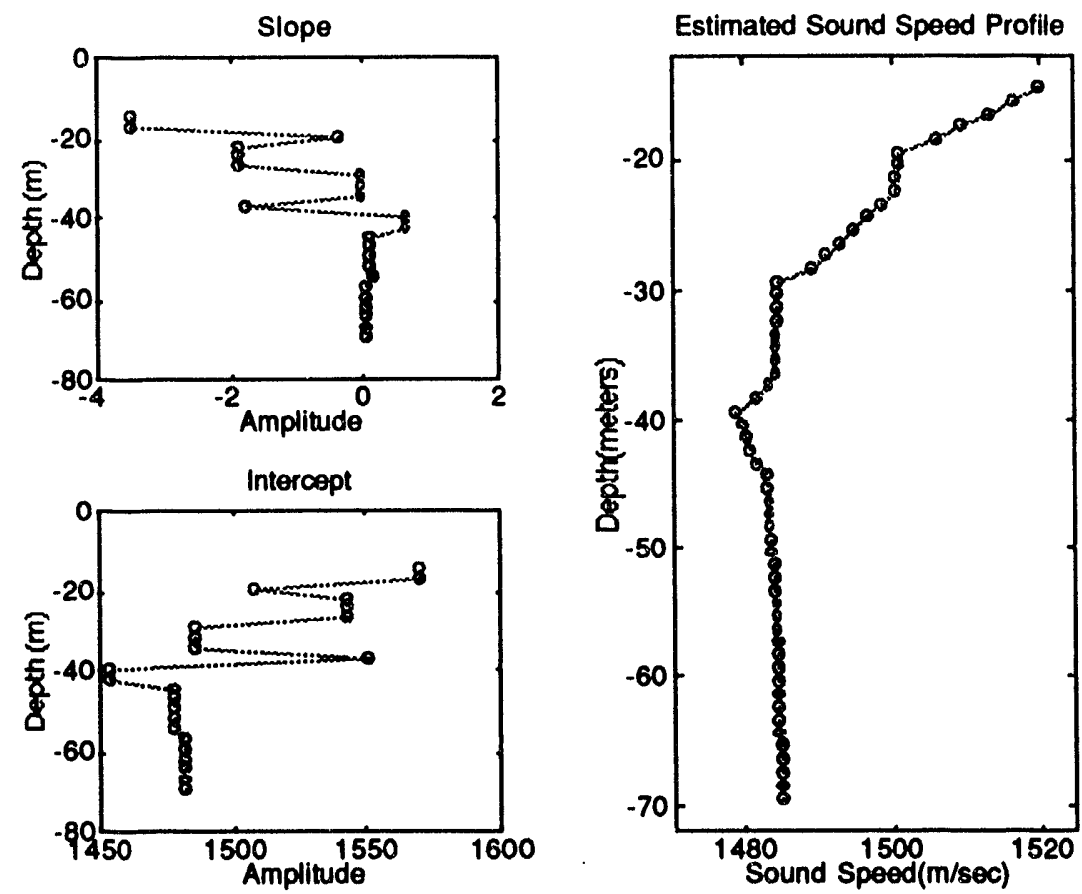

Figure 1. Model-Based Inversion: (a) Slope Estimation. (b) Intercept Estimation (c) Sound Speed Estimation. 

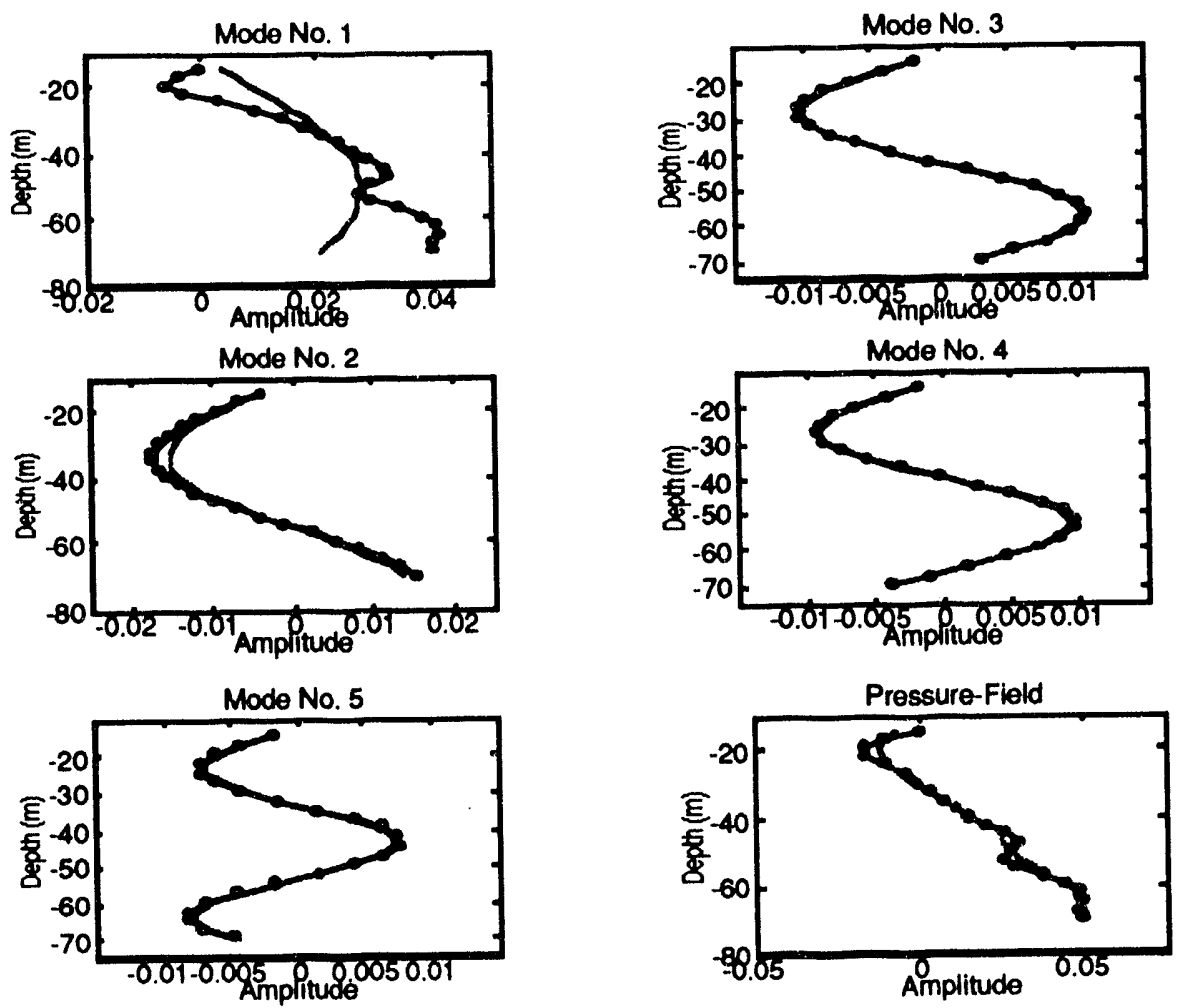

Figure 2. Model-Based Enhancement: (a) Est. Modes 1 \& 3. (b) Est. Modes 2 \& 4. (c) Est. Mode5 \& Pressure-Field
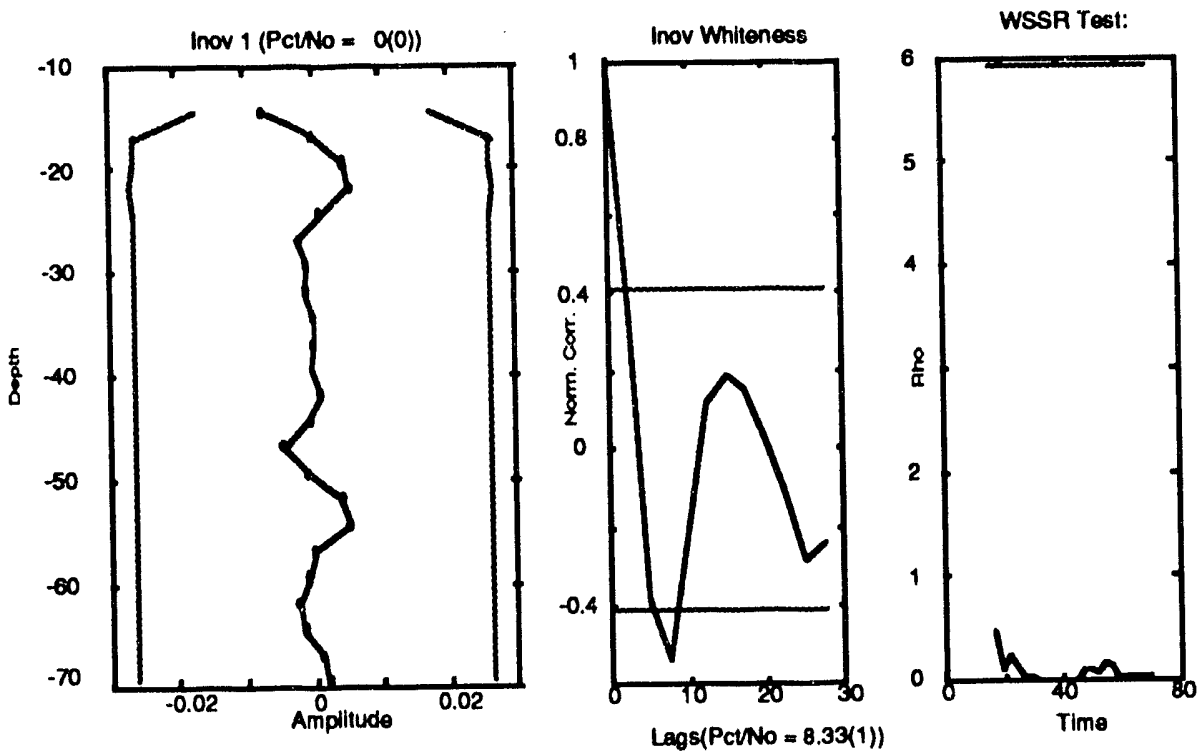

Figure 3. Model-Based Residuals: (a) Innovation/Residual. (b) Whiteness Test (8.3\% Out). (c) WSSR Test (Passed). 

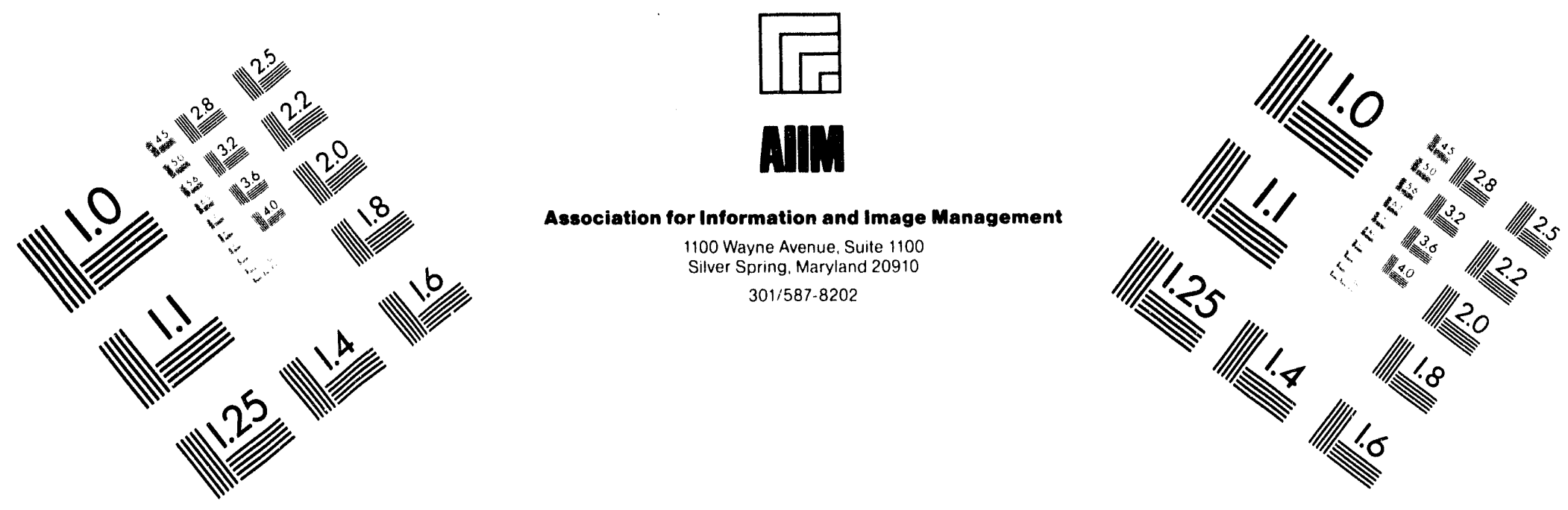

\section{Centimeter}

| Inches
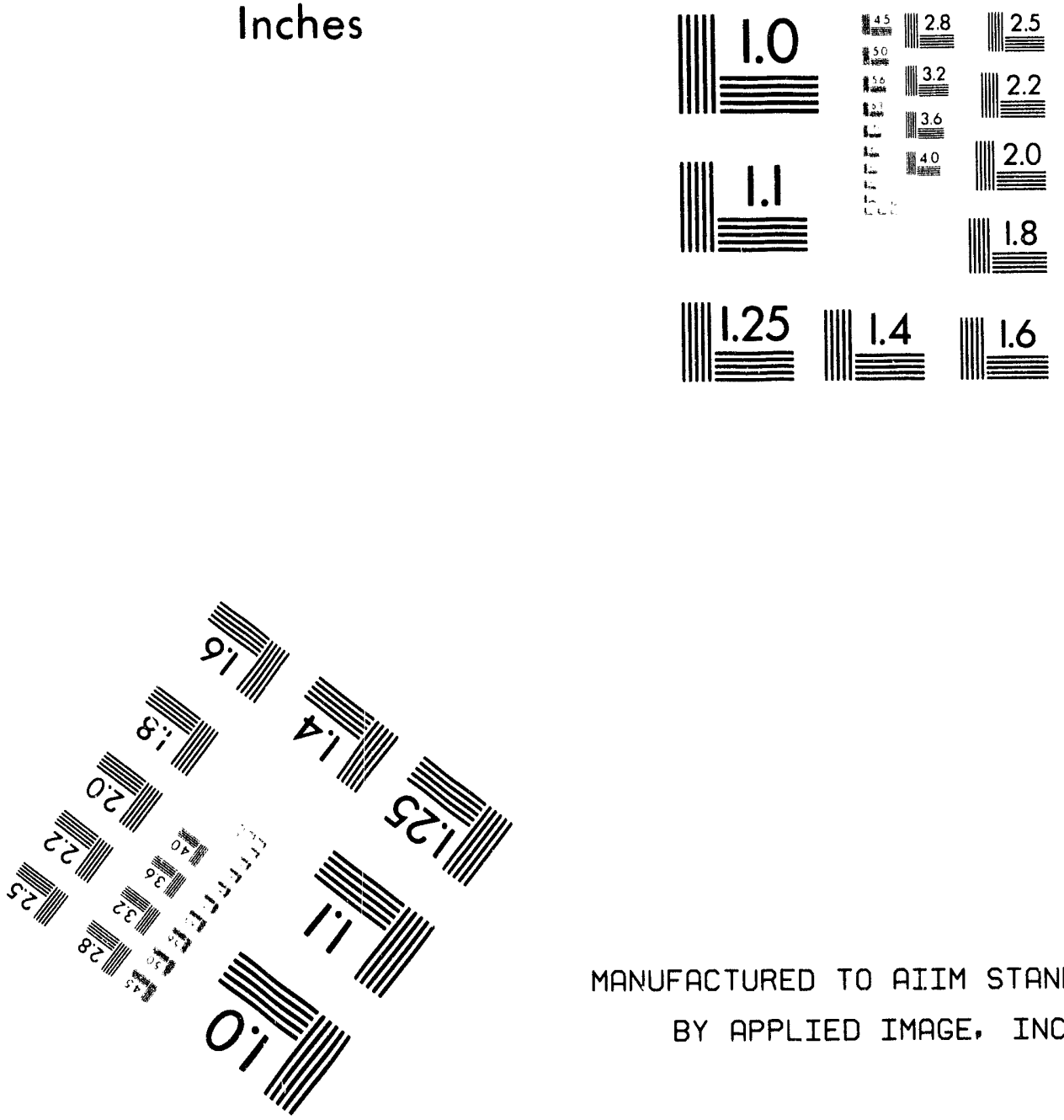

MANUFACTURED TO AIIM STANDARDS

BY APPLIED IMAGE, INC.

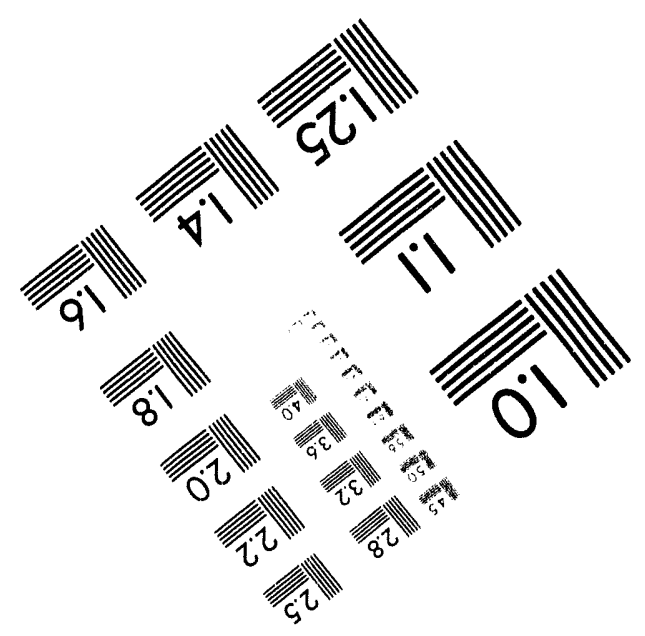




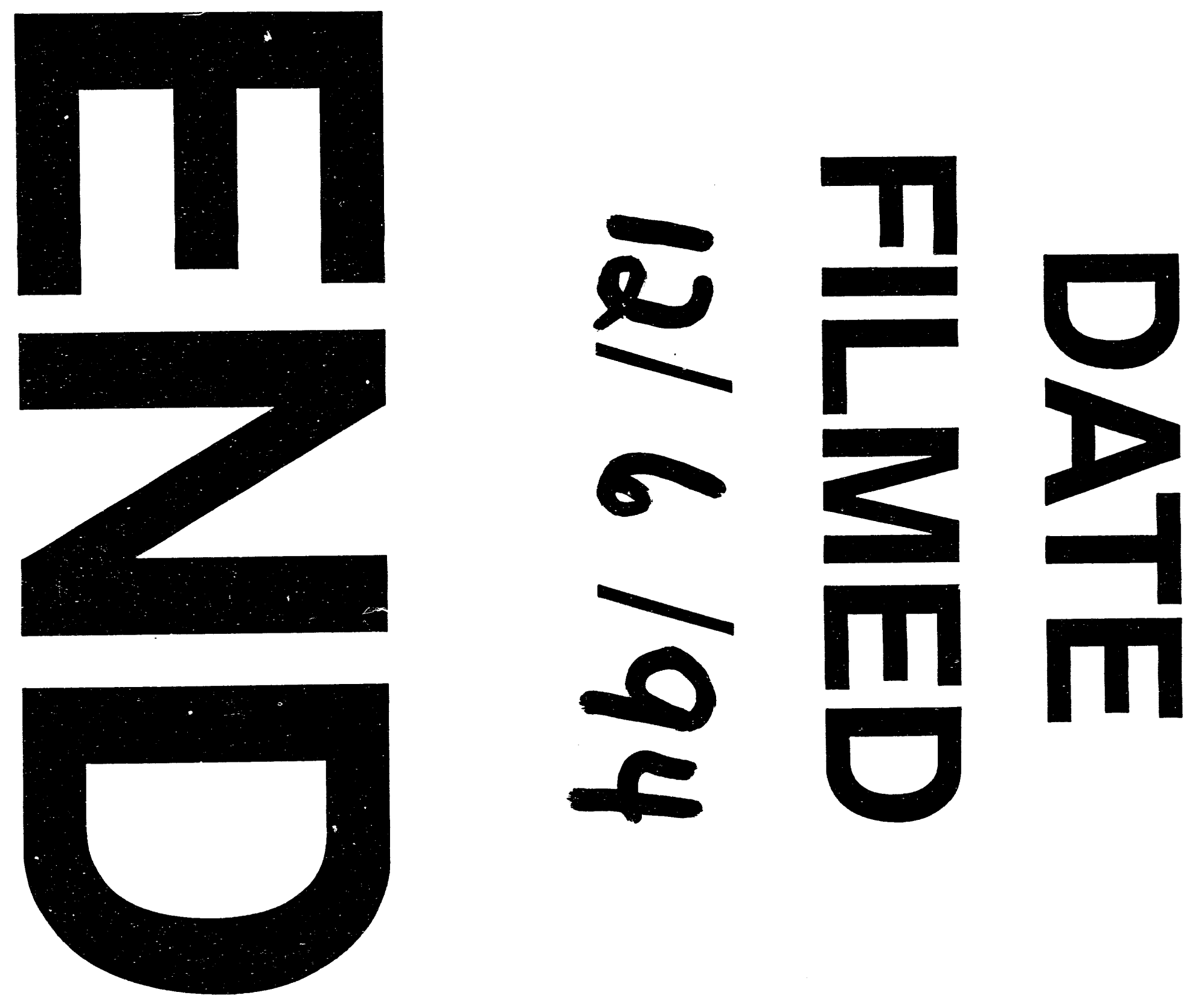